Ana Francisca Romero de Monfrino

\title{
Gestión investigativa en la era del conocimiento
}

\author{
Investigative management in the era of knowledge
}

\author{
Ana Francisca Romero de Monfrino \\ Aromerogm27@gmail.com \\ Universidad Bolivariana de Venezuela \\ Venezuela
}

Recibido: $10 / 07 / 2017$

Aprobado: 25/08/2017

\begin{abstract}
RESUMEN
La intención de este artículo documental es la revisión de diversos enfoques teóricos en cuanto a la gestión investigativa, tomando en cuenta la vinculación universidad comunidad - mundo globalizado, en donde se evidencia que es necesario fomentar una cultura investigativa, formando investigadores que sean capaces de generar el conocimiento con y para la sociedad. Además debe tomarse en cuenta el organizar los procesos de investigación, recordando que las universidades tienen el rol de generar conocimiento científico en pro del desarrollo de la sociedad, formando el talento humano.
\end{abstract}

Palabras clave: formación de investigadores, investigación, gestión investigativa, programas de investigación.

\footnotetext{
ABSTRACT

The intention of this documentary article is the revision of various theoretical approaches in terms of research management, taking into account the link Univarsity - Contexto Community - Society - Globalized World, where there is evidence that it is necessary to promote a culture of research, training researchers that are able to generate knowledge for and to society. In addition, it must take into account the organization of the processes of research, reminding that the universities have the main role of generate scientific knowledge in favour of the development of society, forming the human talent.
} 


\section{CIENCIAMATRIA \\ Revista Interdisciplinaria de Humanidades, Educación, Ciencia y Tecnología \\ Año V. Vol. V. №9. Julio - Diciembre 2019 \\ Hecho el depósito de ley: pp201602FA4721 \\ ISSN-L: 2542-3029; ISSN: 2610-802X \\ Universidad Nacional Experimental Francisco de Miranda (UNEFM). Santa Ana de Coro. Venezuela}

\section{Ana Francisca Romero de Monfrino}

Keywords: training of researchers, research, research management, research program.

\section{INTRODUCCIÓN}

Las universidades juegan un papel importante en la configuración de la sociedad dada su alta responsabilidad en la formación de la humanidad y en la generación del conocimiento científico. En los países en vía de desarrollo se concibe cada día más a la universidad, como un agente clave para el desarrollo de potencialidades, recursos y valores para la sociedad, y es considerada como una realidad compleja capaz de generar riqueza y capital humano, por lo tanto su vinculación al entorno se traduce en la cooperación del desarrollo y competitividad regional, y está continuamente afectada por los cambios que pueden ser políticos, económicos, sociales y culturales.

Es por ello que nuestro país necesita una verdadera transformación universitaria no solo en el campo de la educación, sino también de la investigación científica, en aplicar la ciencia y la tecnología al desarrollo de la nación, el conocimiento generado debe estar vinculado a la construcción de la sociedad, y por lo tanto a la realidad de las comunidades. En este sentido es pertinente tener en consideración lo planteado por (Noriero - Escalante y Victorino-Ramírez, 2019) al indicar que

Hay que señalar que al interior de las universidades también existen grupos de poder $e$ inercias institucionales que frenan las posibilidades de transformar la educación hacia un plano de excelencia académica y con sentido público, crítico, autónomo, democrático y comprometido socialmente. (p. 164).

Las instituciones universitarias deben realizar investigaciones como verdaderos procesos de generación de conocimiento con la finalidad de transcender posiciones de confort, para que sean capaz de crear destrezas metodológicas y formar investigadores, por lo que desde las tesis de grados, los trabajos de ascensos hasta asignaciones en las unidades curriculares deben ser considerados elementos de un 
sistema organizacional conducido a la investigación, a la generación de productos de conocimiento destinados a algún ámbito de consumo -la sociedad- (Padrón, 1994).

Por su parte, la Organización de las Naciones Unidas para la Educación, la Ciencia y la Cultura (UNESCO, 1998) menciona que una de las misiones de la educación superior es la de promover, generar y difundir conocimientos por medio de la investigación y, como parte de los servicios que ha de prestar a la comunidad, proporcionar las competencias técnicas adecuadas para contribuir al desarrollo cultural, social y económico de las sociedades, fomentando, desarrollando la investigación científica y tecnológica a la par que la investigación en el campo de las ciencias sociales.

El hombre se encuentra en una constante búsqueda de explicaciones, transformaciones de su propia realidad, que conlleva a una continua producción de conocimiento y desarrollo científico a diario, y que no todos son socializados, quedando en las gavetas y archivos de las universidades, sin aprovechar todas las potencialidades del conocimiento generado por los profesionales, hasta reprimiendo los que se pueden generar partiendo de la socialización de los mismos.

Entonces ¿Será necesaria la gestión investigativa, en la era del conocimiento, de la información, y en un mundo globalizado como el actual? Esta inquietud se la han realizado diferentes investigadores que han comenzado a trabajar en cómo darle una explicación científica y cuáles serían las estrategias a utilizar, para que se alcancen los objetivos de generar conocimiento de una manera eficiente en lo que se puede ver como tres etapas: la generación, la sistematización y socialización, donde se evidencie el desarrollo de los profesionales y de la sociedad.

\section{DESARROLLO}

A nivel mundial la educación universitaria, es pilar fundamental del desarrollo cultural, social y económico. Por lo que constantemente se tiene la necesidad de implantar desafíos que implican una transformación permanente, con la finalidad de presentar soluciones a la profunda crisis de valores que vive la sociedad contemporánea. En 


\section{CIENCIAMATRIA \\ Revista Interdisciplinaria de Humanidades, Educación, Ciencia y Tecnología \\ Año V. Vol. V. №9. Julio - Diciembre 2019 \\ Hecho el depósito de ley: pp201602FA4721 \\ ISSN-L: 2542-3029; ISSN: 2610-802X \\ Universidad Nacional Experimental Francisco de Miranda (UNEFM). Santa Ana de Coro. Venezuela}

\section{Ana Francisca Romero de Monfrino}

donde nuestro país no está excepto, de hecho mi criterio personal es, que estamos viviendo actualmente una de las mayores crisis de valores en nuestra sociedad.

Sierra, y col. (2010), expresaron que es evidente el papel que juega la universidad como eje central y motor, de la llamada industria del conocimiento, en la que este se convierte en activo principal desde dos perspectivas: la de insumo y la del producto final.

Lo que da paso a los llamados Programa de Investigación Científica, que de acuerdo con Lakatos (1978) no son más que un equipo con grandes logros científicos, considerados también como Unidad de Análisis Epistemológica constituida por una secuencia de teorías científicas con continuidad espacio-temporal que relaciona a sus miembros, estableciéndose versiones modificadas según un plan inicial común.

Si se observa la investigación desde el punto de vista gerencial, estos no pueden tener exclusivamente una descripción metodológica de tipo lógico-estructural, sino que también una descripción organizativo-gerencial, referida a las condiciones de productividad, es decir, efectividad de los procesos frente a las necesidades y oportunidades -realidad y contexto estudiado-.

En este mismo orden de ideas, la gerencia de la investigación tiene dos componentes que según Castañeda y Castañeda (2007) son la gerencia y la investigación, entendiendo como gerencia el arte y la ciencia de trabajar en equipo para el logro de los objetivos de una organización (institución), sin olvidar sus funciones básicas planificación, organización, dirección y control. Por su parte la investigación es un procedimiento reflexivo, sistemático, controlado y crítico que tiene como finalidad describir e interpretar los hechos o fenómenos, relaciones y leyes de un determinado ámbito de la realidad (Ander-egg, 1992).

Las universidades deben fortalecer la promoción, generación y difusión del conocimiento mediante la investigación. Como organizaciones tiene el compromiso de ser fuertes desde el punto de vista de su estructura académico-científica y de su capacidad técnica gerencial, lo que la obliga a contar con un capital humano, científico y 


\section{CIENCIAMATRIA \\ Revista Interdisciplinaria de Humanidades, Educación, Ciencia y Tecnología \\ Año V. Vol. V. №9. Julio - Diciembre 2019 \\ Hecho el depósito de ley: pp201602FA4721 \\ ISSN-L: 2542-3029; ISSN: 2610-802X \\ Universidad Nacional Experimental Francisco de Miranda (UNEFM). Santa Ana de Coro. Venezuela}

\section{Ana Francisca Romero de Monfrino}

tecnológico situado en los más altos estándares de eficiencia para gestionar una academia con calidad, ya que de ello depende el desarrollo económico y social de las regiones (Sierra, Nair, Rangel, y Alvarado, 2010).

Según el documento de la UNESCO (1995) Políticas para el cambio y desarrollo de la Educación Superior: "la clave para poner en marcha los procesos necesarios para enfrentar los desafíos del mundo moderno y, mediante sus funciones de docencia, extensión, investigación, representa el factor necesario en la transformación y aplicación de estrategias y políticas de desarrollo".

En la sociedad futura el recurso clave será el conocimiento y sus trabajadores serán el grupo dominante de la población activa. Estos cambios fundamentales inciden en la estructura de la sociedad y crean una nueva dinámica social, económica y política (Drucker 1994). En ese sentido los líderes de las universidades, además de poseer un alto nivel de formación disciplinar, deben desarrollar e implementar herramientas gerenciales que le garanticen un grado sumo de cumplimiento de las demandas sociales del entorno. Hecho que se puede lograr con la investigación con pertinencia social.

González y Castillo (2011) sostienen que es necesario propiciar la creación de conocimiento, como política proactiva, propiciando una serie de garantías laborales y académicas para que docentes y estudiantes desarrollen a plenitud la labor investigativa. Promover la inter y trandisciplinariedad, de tal manera que se aborden investigaciones desde los diferentes enfoques. El conocimiento creado debe ser socializado, además de impulsar las redes de investigación para que se amplíen sus fronteras de lo regional a lo nacional y luego a lo internacional.

Es por ello que es necesaria la gestión de la investigación universitaria donde las nuevas generaciones deben ver las investigaciones como un proyecto de vida, insertándose en el desarrollo integral de las comunidades, formar conciencia autónoma a los valores, de libertad, justicia y solidaridad, como expresan Ferrer y Clemenza 


\section{CIENCIAMATRIA \\ Revista Interdisciplinaria de Humanidades, Educación, Ciencia y Tecnología \\ Año V. Vol. V. №9. Julio - Diciembre 2019 \\ Hecho el depósito de ley: pp201602FA4721 \\ ISSN-L: 2542-3029; ISSN: 2610-802X \\ Universidad Nacional Experimental Francisco de Miranda (UNEFM). Santa Ana de Coro. Venezuela}

\section{Ana Francisca Romero de Monfrino}

(2006) se hace indispensable lograr una correlación entre la generación de beneficios a la sociedad y la generación de conocimiento.

Adicionalmente, la dinámica investigativa dentro de los grupos de investigación o programas de investigación, requiere de un proceso organizacional y gerencial, que les permita a los investigadores y co-investigadores adaptarlas de acuerdo con sus necesidades, intereses y entorno donde se encuentre.

Entonces si hablamos de gerencia de la investigacion las funciones principales que se ejecutaran según Castañeda y Castañeda (2007) son las siguientes:

Planificación, toda investigación debe estar en inscritas en un programa o línea de investigación, además debe definir su alcance, los objetivos, políticas y estrategias de financiamiento.

Organización, durante el desarrollo de la investigación se distribuye funciones y tareas, se establecen y desarrollan redes, asigna y coordina tecnología de información, talento humanos, recursos materiales, espacios y condiciones idóneas para fomentar la creación y difusión de conocimiento.

Dirección, se ejecuta en función del liderazgo para la construcción del conocimiento y desarrollo de capacidades creativas e innovadoras de los seres humanos.

Control, no solo evaluando el logro de los objetivos planteados, sino que también en términos de eficiencia del máximo bienestar humano al menor costo posible y de efectividad 0 impacto social, bajo una concepción pluridisciplinaria y transdisciplinaria. Donde se tenga una participación activa, se tenga un cambio y un desarrollo de la realidad.

Para Gil, y col. (2012) se deben crear sistemas innovadores de gestión de la investigación, que permitan sistematizar la canalización de las iniciativas de los investigadores, la captación de recursos y la transferencia de los resultados de la investigación. En este sentido, es recomendable el desarrollo de un modelo en red. 


\section{Ana Francisca Romero de Monfrino}

Las universidades en los últimos años se han dedicado a formar cuadros de profesionales dándole mayor valor a la gestión de la docencia en las universidades, dejando de lado la gestión de investigación. Entre las razones, está la poca tradición científica de nuestros países, la presión de la demanda social por matrícula estudiantil, carencia de un claro concepto del deber ser de las universidades por parte de profesores y estudiantes y en particular, de la alta gerencia universitaria preocupados por resolver lo inmediato pero dejando de lado en algunos casos, la razón de ser de las instituciones (Ferrer y Clemenza, 2006).

Lo anteriormente mencionado, se percibe con mucha frecuencia en la realización de los trabajos de grado de los estudiantes, y en todas las actividades investigativas que se desarrolla; existen investigaciones enfocadas a áreas donde los docentes no poseen competencias, ni hay un lineamiento claro y preciso que guíe la producción ni la aplicación de conocimiento que puede generarse de dicha investigación (Fuenmayor, Perozo, y Narvaéz, 2007).

Al contrario la investigación debe ser vista como acción global, la producción de conocimientos es considerada como un hecho organizacional, como una actividad que sólo es rendidora en la medida en que los trabajos parciales e individuales se agrupen alrededor de sistemas de objetivos, planes, tecnologías, estructuras de procesos, controles, vías de alimentación-suministro, entre otros, todo enmarcado en significativos análisis de las demandas sociales (Padrón, 1994). Por su parte Johnson y Johnson (1993) y Colle (1991) citados por Chacín y Briceño (1995) y Fuenmayor, Perozo, y Narvaéz (2007) expresan que el trabajo en equipo no es una simple técnica de dinámica de grupos ni una decisión voluntarista, sino un proceso de aprendizaje que promueve y estimula la creatividad y la conformación de comunidades críticas y autoreflexivas.

Lo cual Lakatos (1983) Ilamo -programa de investigación- que no son más que un conjunto borroso de trabajos investigativos individuales, ordenado por una relación de secuencia temporal y, además, por relaciones de inclusividad y complementaridad. 


\section{CIENCIAMATRIA \\ Revista Interdisciplinaria de Humanidades, Educación, Ciencia y Tecnología \\ Año V. Vol. V. №9. Julio - Diciembre 2019 \\ Hecho el depósito de ley: pp201602FA4721 \\ ISSN-L: 2542-3029; ISSN: 2610-802X \\ Universidad Nacional Experimental Francisco de Miranda (UNEFM). Santa Ana de Coro. Venezuela}

\section{Ana Francisca Romero de Monfrino}

Desde el punto de vista de su estructura diacrónica, entonces, la investigación es considerada en términos de Programas o redes problemáticas y no como hechos individuales aislados entre sí.

La investigación requiere de un trabajo en equipo y de cooperación, para alcanzar la mayor productividad en sus objetivos trazados, y expandir el desarrollo de las líneas y redes donde está inmersa. Por lo cual se considera urgente que las instituciones educativas, estimulen la conformación de equipos interdisciplinarios de investigación, capaces de abordar, en forma conjunta, estudios de diferentes niveles de complejidad, conservando autonomía e independencia (Fuenmayor, Perozo, y Narvaéz, 2007).

Son pocos los equipos de trabajo amplios, interdisciplinarios, realmente integrados, donde se aprovecha al máximo la experiencia individual de cada miembro sin que sea afectado si estos miembros cambian. Dichos equipos son organizados alrededor de grandes problemas, sin problemas de prestigio, jerarquía e internacionales (Varsavsky, 2006).

La gestión de la investigación debe fomentar la creación y consolidación grupos de investigación multidisciplinarios, productivos o sociales, de relevancia para las comunidades o la nación. González y Castillo (2011) hacen referenca a ciertos aspectos claves para la labor de los grupos de investigación como por ejemplo el desarrollo de líneas de investigación estratégicas, investigadores en diferentes áreas investigaciones multidisciplinarias-, evitar el divorcio entre las instituciones, intercambio de conocimiento con otras comunidades científicas, creación de redes, entre otros Adicionalmente, se debe incrementar vínculos con los sectores públicos y privados para la detección de temas más relevantes presentes y futuro de ambos sectores, además de obtener financiamiento, sin olvidar las realidades de las comunidades. En este mismo orden de ideas, para la UNESCO citado por (Pesca de Acosta, 2012) la universidad ha de aplicar capacidad prospectiva y anticipatorio que le permita, al iluminar posibles alternativas y escenarios, construir la mejor opción posible de futuro. 


\section{CIENCIAMATRIA \\ Revista Interdisciplinaria de Humanidades, Educación, Ciencia y Tecnología \\ Año V. Vol. V. №9. Julio - Diciembre 2019 \\ Hecho el depósito de ley: pp201602FA4721 \\ ISSN-L: 2542-3029; ISSN: 2610-802X \\ Universidad Nacional Experimental Francisco de Miranda (UNEFM). Santa Ana de Coro. Venezuela}

\section{Ana Francisca Romero de Monfrino}

La docencia y la investigación universitaria tienen una finalidad social y política bien definida, deben capaces de contribuir a la búsqueda investigativa en la necesidad de resolver problemas básicos para nuestra sociedad (Ferrer y Clemenza, 2006). Las investigaciones universitarias deben ser auténticos procesos de producción de conocimientos y no como mero ejercicio curricular que sólo permite, en el mejor de los casos, crear destrezas metodológicas, teniendo en cuanta que es necesario formar verdaderos investigadores.

Los criterios pedagógicos centrales de Varsavsky (2006) hace mención a que toda enseñanza - formación desde estar ligada al proceso productivo de la nacion, es necesaria la participacion activa del estudiante en tareas productivas y de investigacion normales.

En la gestión investigativa se debe ser capaz de analizar el mercado, de diseñar redes de problemas investigativos, de distribuir a estudiantes y docentes alrededor de las funciones y tareas planteadas según esas redes problemáticas, de asignar recursos y asistencia técnica, de promover y difundir los productos logrados y, finalmente, de realimentar el sistema, que Padrón (1994) hace referencia como la concepción gerencial de la investigación. Donde es necesaria la formación continua de docentes y directivos del sistema educativo general es decir desde el nivel básico hasta el universitario, como docente investigador, el cual cultive la capacidad de reflexión permanente en la acción y sobre la acción, para lograr la transformación de la realidad.

Para Padrón (1994) la subsiguiente tarea de una gerencia de investigaciones consiste, entonces, en ofrecer a estudiantes y docentes los programas de investigación previamente diseñados, en organizarlos alrededor de esos programas, en brindarles asistencia técnico-metodológica y en controlar las ejecuciones individuales. Es decir es necesario aprender-a-investigar" donde las investigaciones realizadas por los estudiantes y por los docentes no sean solo un objeto de evaluación, sino un producto que puede ser aprovechado dentro de algún plan global de investigación. 


\section{CIENCIAMATRIA \\ Revista Interdisciplinaria de Humanidades, Educación, Ciencia y Tecnología \\ Año V. Vol. V. №9. Julio - Diciembre 2019 \\ Hecho el depósito de ley: pp201602FA4721 \\ ISSN-L: 2542-3029; ISSN: 2610-802X \\ Universidad Nacional Experimental Francisco de Miranda (UNEFM). Santa Ana de Coro. Venezuela}

\section{Ana Francisca Romero de Monfrino}

Por esta razón la Universidad debe concebirse como un espacio creativo de aprendizaje, de investigación, de innovación, no sólo para satisfacer los requerimientos académicos y científicos sino para promover respuestas contundentes a los problemas que afectan la realidad y donde el espíritu innovador para abordar la solución de dichos problemas, será el camino de la permanente búsqueda de respuestas que interrumpen la vida de la sociedad.

La universidad debe formar profesionales de mentalidades solidarias y creativas (Varsavsky, 2006). En la actualidad las investigaciones deben proporcionar a la sociedad no sólo el saber cómo (Know how) sino además el saber por qué o para qué (Know why).

Como se ha evidenciado la sociedad demanda con más fuerza de las universidades, la formación de profesionales competentes, con formación integral, la que no solo depende de los conocimientos y habilidades que se adquieren en los claustros docentes sino de las convicciones, sentimientos y valores éticos que regulan la actuación profesional del egresado, lo cual requiere una sólida formación axiológica. Articulado la investigación con las otras funciones universitarias brinde a la sociedad la oportunidad de contar con innovaciones de productos y de procesos (Gil y col, 2012).

La legitimidad social de la investigación universitaria está relacionada con el deber que tiene la Universidad de concebir el proceso investigativo como una construcción social que se realiza en un contexto espacio-temporal condicionado por circunstancias históricas, sociales y psicológicas provenientes de contextos internacionales, nacionales y locales (Ordoñez, 2008).

El reto de la investigación es impulsar el conocimiento a fin de hacer frente a los cambios de la sociedad actual, exigiendo una labor constante en materia de gestión social del conocimiento (Castañeda y Castañeda, 2007).

Para Varsavsky (2006) lo que se investiga en una sociedad es lo que la sociedad considera suficientemente importante. Dicha importancia no tiene nada que ver con la capacidad de respuesta, sino por las caracteristicas ideologicas. Es necesaria la 


\section{CIENCIAMATRIA \\ Revista Interdisciplinaria de Humanidades, Educación, Ciencia y Tecnología \\ Año V. Vol. V. №9. Julio - Diciembre 2019 \\ Hecho el depósito de ley: pp201602FA4721 \\ ISSN-L: 2542-3029; ISSN: 2610-802X \\ Universidad Nacional Experimental Francisco de Miranda (UNEFM). Santa Ana de Coro. Venezuela}

\section{Ana Francisca Romero de Monfrino}

vinculacion formacion - investigacion - comunidad para provocar transformaciones en la realidad (Cordova, s.f.).

\section{CONCLUSIÓN}

La gestión investigativa en la era del conocimiento debe contribuir al aprovechamiento y socialización de los saberes de los profesionales, propiciar la formación y desarrollo del sujeto, individual y colectiva, inter y transdisciplinaria. Las universidades deben superar su carácter pedagógico (docencia) y entrar a la sociedad del conocimiento (generación de conocimiento).

Estamos en una sociedad en la que el desarrollo socioeconómico se vincula cada vez más con el conocimiento, que debe ser útil y relevante, vinculando la comunidaduniversidad-sector público y privado. Teniendo claro, que cualquier orientación de gestión de la investigación universitaria, deberá estar inserta en planes locales, regionales y nacionales, en cuya formulación está integrada la comunidad científica.

En las instituciones universitarias las políticas deben girar en pro de fomentar una cultura investigativa en los estudiantes, docentes incluyendo al personal administrativo y obrero, capaz de generar conocimiento con cada actividad que se realice, sin que sea vista como un producto necesario para conseguir una calificación, un título o su ascenso, sino como una forma de generar conocimiento propiamente dicha.

Adicionalmente se deben romper las barreras de las instituciones, trazar vínculos con las comunidades y sociedades científicas con la finalidad de dar respuestas a problemáticas específicas y crear redes entre las diferentes instituciones, y difundir el conocimiento generado, ya sea por medio de publicaciones o por disertaciones en actividades científicas.

No podemos olvidar que estamos en una era donde el poder lo tienen quien tiene el conocimiento, por lo cual en cada universidad, institución y organización del mundo, cada día se está en la búsqueda de nuevas innovaciones que generen conocimiento, lo 


\section{Ana Francisca Romero de Monfrino}

que hace necesaria la gestión investigativa, en la era del conocimiento, de la información, y en un mundo globalizado como el actual.

\section{REFERENCIAS CONSULTADAS}

1. Ander-egg, E. (1992). Tecnicas de investigacion social. Buenos Aires: Humanistas.

2. Castañeda, G., \& Castañeda, R. (2007). Gerencia de investigacion: Criterios gerenciales aplicados a la investigacion. Orbis / Ciencias humanas, II(6), 18-47.

3. Cordova, M. (s.f.). Integracion Socioeducativa como eje estrategico para la transformacion universitaria. Caracas: CNU - OPSU - UBV.

4. Cova, A., Inciarte, A., \& Prieto, M. (2005). Lakatos y los programas de investigación científica. Omnia, 11(3), 83-108.

5. Ferrer, J., \& Clemenza, C. (2006). Gestión de la investigación universitaria: Un paradigma no concluido. Multiciencias, VI(2), 188 - 193.

6. Fuenmayor, B., Perozo, S., \& Narvaéz, J. (mayo-agosto de 2007). Investigación y gestión del conocimiento. Caso: Instituto Universitario de Tecnología de Cabimas. Laurus, XIII(24), 355-376.

7. Gil, J., Dominguez, R., Garcia, L., Mathison, L., \& Gandara, J. (2012). La investigaci'on universitaria como eje de la transferencia social del conocimiento. Ciencias y Tecnolog'ıa, VI(1), 41-51.

8. González, J., \& Castillo, B. (2011). La gestión investigativa universitaria: un problema de vieja data sin pronta solución (Caso analítico uptC). Económicas CUC, 32(1), 9-34.

9. Lakatos, I (1978) La metodología de los programas de investigación científica. Alianza editoral.

10. Noriero-Escalante, L., \& Victorino-Ramírez, L. (2019). Balance crítico del Plan Institucional de Desarrollo 2009-2025 de la Universidad Autónoma Chapingo Perspectivas de su contenido, alcances y limitaciones. Revista Arbitrada Interdisciplinaria Koinonía, 4(7), 147-167. Recuperado de http://fundacionkoinonia.com.ve/ojs/index.php/revistakoinonia/article/view/198/16 2 


\section{CIENCIAMATRIA \\ Revista Interdisciplinaria de Humanidades, Educación, Ciencia y Tecnología \\ Año V. Vol. V. Nº9. Julio - Diciembre 2019 \\ Hecho el depósito de ley: pp201602FA4721 \\ ISSN-L: 2542-3029; ISSN: 2610-802X \\ Universidad Nacional Experimental Francisco de Miranda (UNEFM). Santa Ana de Coro. Venezuela}

\section{Ana Francisca Romero de Monfrino}

11. Ordoñez, B. (2008). Estructura diacrónica y sincrónica de los procesos investigativos y legitimidad social de la investigación universitaria. Gestión y Gerencia, II(1), 4-21.

12.Padrón, J. (1994). Organización-gerencia de investigaciones y estructuras investigativas. Universitas 2000, 18 (3-4), 109-132.

13. Pesca de Acosta , C. (2012). Educación universitaria en desarrollo y aplicación del talento humano y la gestión del conocimiento. Intersedes, XIII(25), 98-115.

14. Sierra, W., Nair, A., Rangel, O., \& Alvarado, M. (2010). Liderazgo moderno y tendencias gerenciales: cambios paradigmaticos en la gestion de la univerisdad como empresa del conocimeiento. Revista Cientifica Guillermo de Odkam, 8(2), 11-22.

15. UNESCO. (1995). Política para el cambio y el desarrollo de la educación superior. Quito, Ecuador.

16. UNESCO. (2009). Conferencia Mundial sobre la Educación Superior - 2009: La nueva dinámica de la educación superior y la investigación para el cambio social y el desarrollo. COMUNICADO, Paris. Recuperado el 12 de abril de 2016, de http://www.unesco.org/education/WCHE2009/comunicado_es.pdf

17. Varsavsky, O. (2006). Hacia una politica cientifica nacional (1era ed.). Caracas, Venezuela: Monte Avila Editores Latinoamericana C.A.

C2019 por el autor. Este artículo es de acceso abierto y distribuido según los términos y condiciones de la licencia Creative Commons Atribución-NoComercial-Compartirlgual 4.0 Internacional (CC BY-NC-SA 4.0) (https://creativecommons.org/licenses/by-nc-sa/4.0/). 